\title{
Dielectric Properties of Liquid Crystal Polymer Substrates in the Region from 90 to $140 \mathrm{GHz}$
}

\author{
Rongxin Mao ${ }^{1}$, Guozhen Zhang ${ }^{1}$, Lu Xu ${ }^{1}$, Haoyu Gao ${ }^{1}$, Jun Yang ${ }^{1,2, *(\mathbb{D}}$, Zhiping Yin ${ }^{1} \mathbb{D}$, Guangsheng Deng ${ }^{3}(\mathbb{D}$ \\ and Hongbo Lu ${ }^{1}$
}

check for updates

Citation: Mao, R.; Zhang, G.; Xu, L.; Gao, H.; Yang, J.; Yin, Z.; Deng, G.;

Lu, H. Dielectric Properties of Liquid Crystal Polymer Substrates in the Region from 90 to $140 \mathrm{GHz}$. Crystals 2022, 12, 170. https://doi.org/ $10.3390 /$ cryst12020170 Academic Editor: Ingo Dierking

Received: 22 December 2021 Accepted: 23 January 2022 Published: 25 January 2022

Publisher's Note: MDPI stays neutral with regard to jurisdictional claims in published maps and institutional affiliations.

Copyright: () 2022 by the authors. Licensee MDPI, Basel, Switzerland. This article is an open access article distributed under the terms and conditions of the Creative Commons Attribution (CC BY) license (https:// creativecommons.org/licenses/by/ $4.0 /)$.
1 Special Display and Imaging Technology Innovation Center of Anhui Province, Academy of Opto-Electric Technology, Hefei University of Technology, Hefei 230009, China; mrx0122@163.com (R.M.); guozhenzhang_mail@163.com (G.Z.); xulu_mail@126.com (L.X.); gaohaoyu5611@163.com (H.G.); zpyin@hfut.edu.cn (Z.Y.); bozhilu@hfut.edu.cn (H.L.)

2 Guangxi Key Laboratory of Wireless Wideband Communication and Signal Processing, Guilin University of Electronic Technology, Guilin 541004, China

3 Anhui Province Key Laboratory of Measuring Theory and Precision Instrument, School of Instrument Science and Optoelectronics Engineering, Hefei University of Technology, Hefei 230009, China; dgsh@hfut.edu.cn

* Correspondence: junyang@hfut.edu.cn

\begin{abstract}
In this article, we present a study of the equivalent dielectric constant of two nematic liquid crystals (LCs) with different thicknesses, in the region from 90 to $140 \mathrm{GHz}$. The equivalent dielectric constant of the LCs was measured using a frequency selective surface (FSS). The LC-based tunable FSS with $22 \times 25$ unit cells was printed on a quartz substrate with an area of $4 \times 4 \mathrm{~cm}^{2}$ and a thickness of $480 \mu \mathrm{m}$; the LC layer with thicknesses ranging from 30 to $100 \mu \mathrm{m}$ acted as a substrate. The FSS featured a maximum frequency-shifting range of $8.15 \mathrm{GHz}$ and $30 \mu \mathrm{m}$-thick LC layers with mutually orthogonal rubbing directions were deposited on it. The results show that the initial equivalent dielectric constant of the LC layer increased with the LC layer thickness, while the saturationequivalent dielectric constant remained almost constant. This work provides LC parameters that can be useful for the design of LC-based devices in the millimeter and terahertz ranges.
\end{abstract}

Keywords: nematic liquid crystal; frequency selective surface; electrical control; dielectric constant

\section{Introduction}

Liquid crystals (LCs) feature the fluidity of liquids and the anisotropy of crystals and are considered to be excellent materials for controlling electromagnetic waves. The nematic phase is the simplest LC phase. The long axes of the rod-shaped molecules of this type of LC point in the same direction or nearly parallel to each other. This spontaneous arrangement of molecules with their long axes pointing along the same direction endows the LCs with a high degree of birefringence [1]. In the past few years, the application of LCs has gradually become widespread in the millimeter and terahertz $(\mathrm{THz})$ bands [2-5]. LC-based microwave and THz devices, such as modulators [6], filters [7], absorbers [8], transduces [9,10], and phase shifters [11], can be easily adjusted and controlled by changing the electric field or magnetic field. They offer the advantages of low power consumption and low cost [12]. In order to precisely design LC-based devices, it is essential to accurately investigate the dielectric properties of LCs at THz frequency [13-17]. In 2010, Bulja proposed a broadband planar transmission line method to characterize the physical parameters of the LCs E7, K15, E44, E63, and MDA-00-3506 in the 30-60 GHz millimeter wave frequency range [18]. In 2012, Dickie used a frequency-selective surface (FSS) to accurately measure the dielectric constant and loss tangent of the GT3-23001 and BL037 LCs in the region from 140 to 165 $\mathrm{GHz}$ [19]. In 2015, Deo et al. proposed a simple microstrip structure that can measure the dielectric constant and loss tangent of nematic liquid crystals (NLCs) at 15-65 GHz. In this manner, they were able to obtain the dielectric constant of the NLCs MDA-00-3506 
and GT3-23001 [20]. In 2020, Ji et al. measured and compared the THz birefringence of a polymer-dispersed liquid crystal doped with gold nanoparticles [21]. In 2020, Johri et al. investigated the dielectric properties of PentylCyano Biphenyl NLCs in a temperature range of 297-316 degrees $\mathrm{K}$ at $9.0 \mathrm{GHz}$ and $20.9 \mathrm{GHz}$ frequencies [22]. In the same year, Nova et al. provided an accurate measurement of the complex permittivity of GT3-23002 LC at microwave frequencies [23].

In LC-based tunable devices, the LC layer is typically used as a substrate with adjustable dielectric constant. LCs are composed of rod-shaped molecules, and an external electrical field can be used to align the direction of the LC molecules along the field lines. The tunability of LC polymer substrates is the result of the interaction of a polyimide (Pi) alignment layer with the external electrical field [24]. As the electrode coverage is insufficient and the force is weak away from the Pi alignment layer, the equivalent dielectric constant of thick LC layers can be distinguished from birefringence [25]. However, an accurate model would be able to better study the properties of the target object in applications and provide a more realistic fit [26]. The creation of an accurate model requires an understanding of the specific properties of each material. Not only is it easy to optimize the simulation, but it is also beneficial to obtain accurate test results. It is necessary to research the effect of the thickness of the LC layer on its equivalent dielectric constant for LC-based tunable devices.

In this paper, the effect of the thickness of the LC layer on the equivalent dielectric constant was studied. Due to the difficulty of accurately analyzing the anisotropy and inhomogeneity in the direction of the bias field for the intermediate states, no adequate theory exists to directly calculate the dielectric constant of LCs based on voltage. The equivalent dielectric constant of two LCs was measured using an FSS. An FSS consisting of $22 \times 25$ hollow circle units was fabricated and tested. The values of the equivalent dielectric constant of the LCs SLC103014-200 (S200) and E7 with different thicknesses were calculated by fitting the simulated reflectance spectrum to the experimental spectral response at different voltages. Our work provides more accurate LC parameters for the design of LC-based devices in the millimeter and THz ranges.

\section{Methods}

LC materials feature dielectric anisotropy and exhibit different dielectric properties in different frequency bands. Therefore, the method for testing the dielectric properties of LCs varies in different bands. In the $\mathrm{THz}$ band, the main test methods are $\mathrm{THz}$ time-domain spectroscopy (THz-TDS) and the FSS. Although THz-TDS covers a wide frequency range with high resolution, it requires a high test environment (nitrogen environment), a large test voltage, and complex calculations $[27,28]$. Compared with THz-TDS, the FSS features the advantages of being a noncontact, nondestructive, simple, fast, and highly accurate test method $[29,30]$.

Figure 1a shows the overall structure of the proposed device. The top surface of the bottom quartz substrate is printed with a complete copper surface, which can be used as a ground plane, and the bottom surface of the top quartz substrate is printed with a copper layer with a circular hole pattern. The upper and lower quartz thicknesses, dielectric constant, and loss tangent are $480 \mu \mathrm{m}, \varepsilon=3.78$, and $\tan \delta=0.002$, respectively. As shown in Figure $1 \mathrm{~b}$, the thicknesses of the LC layer and copper layer are $h l c=45 \mu \mathrm{m}$ and $h c=0.5 \mu \mathrm{m}$, respectively, where $h l c$ is controlled by microspheres and the copper is deposited by thermal evaporation. The diameter of the hollow circle is $800 \mu \mathrm{m}$ and the period of the unit-cell is $1600 \mu \mathrm{m}$. Table 1 lists the geometric parameters of the unit-cell structure. 


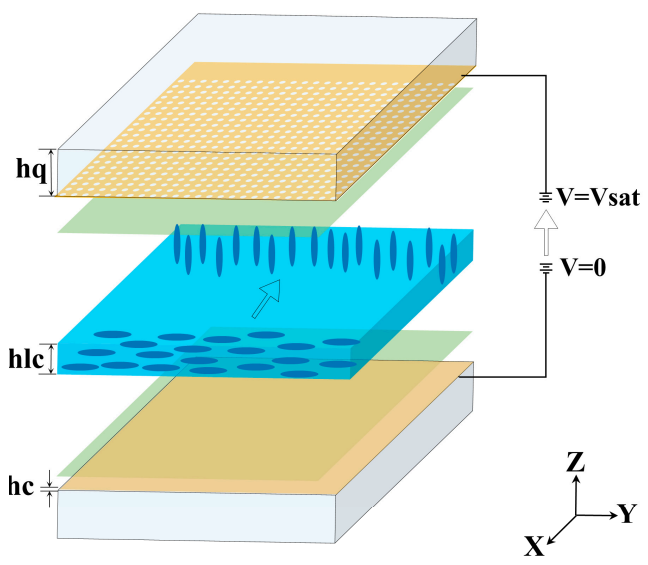

(a)

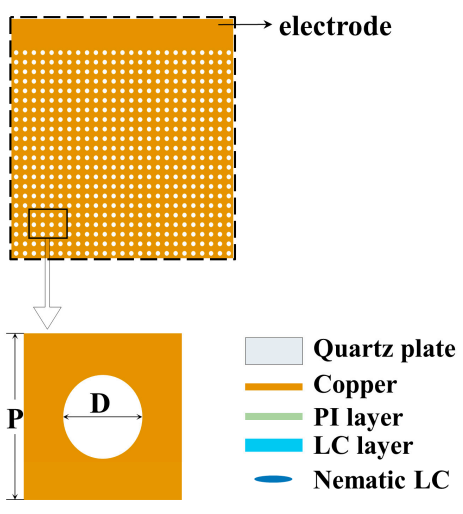

(b)

Figure 1. (a) Unit-cell structure and direction of the LC molecules in two states. (b) Layout of the unit-cell structure with the copper pattern.

Table 1. The parametric dimensions of the FSS structure.

\begin{tabular}{cccccc}
\hline Parameters & $h \boldsymbol{h}$ & $\boldsymbol{h l c}$ & $\boldsymbol{h c}$ & $\boldsymbol{P}$ & $\boldsymbol{D}$ \\
\hline Values $(\mu \mathrm{m})$ & 480 & 45 & 0.5 & 1600 & 800 \\
\hline
\end{tabular}

The bias voltage applied between the two copper layers can generate electric field. Under its influence, the LC molecules point in a different direction, which changes the equivalent dielectric constant of the LC layer. The direction of the LC molecules is not determinate, with no bias voltage. In order to control the direction of the LC molecules, the Pi layers were spun onto the two copper layer. The Pi-covered surface was mechanically rubbed in the direction perpendicular to the electrode. When the applied bias voltage is $0 \mathrm{~V}$, the long axis of the LC molecules is parallel to the surface of each copper layer due to the alignment of the Pi film, where the equivalent dielectric constant of the LC is referred to as $\varepsilon_{\perp}$. As the applied bias voltage reaches the saturation voltage, the long axis of the LC molecules is perpendicular to the surface of the copper layer, and the equivalent dielectric constant of the LC layer reaches the maximum value $\varepsilon_{/ / /}$. Thus, the tunable range of the equivalent dielectric constant of the LC layer is $\Delta \varepsilon=\varepsilon_{/ /}-\varepsilon_{\perp}$. The Finite Element Method (FEM) and periodic boundary condition were used to simulate and optimize the structural parameters to achieve the expected performance. In addition, the FSS features only one distinct absorption peak to facilitate the fit. When the other parameters have been fixed, the resonant frequency point is only decided by the equivalent dielectric constant of the LC layer. By fitting the resonant frequency points of the measured and simulated results, the equivalent dielectric constant of LC layer can be obtained accurately. Of course, as with the equivalent dielectric constant, the equivalent loss tangent also varies with the thickness of the LC layer, but in this study, we mainly focused on dielectric constant.

\section{Experimental Measurements and Results}

A standard photolithography process produced the FSS structure shown in Figure 2a. On the lower surface of the top quartz, a layer of periodic metal metamaterial unit cells was formed by means of exposure, developing, and etching. Figure $2 b$ shows the image of the unit cells of the periodic pattern under a metallographic microscope. Figure $2 \mathrm{c}$ shows the apparatus used for sample testing. The spectral response of the sample was tested by a vector network analyzer (Agilent N5224A), which was accompanied by two horn antennas (with a frequency range of $90-140 \mathrm{GHz}$, a gain of $21 \mathrm{~dB}$ and a $3 \mathrm{~dB}$ bandwidth of 12 degrees) and an F-band module extender (N5262AW08). The $S_{21}$ parameter of the vector network analyzer represents the electromagnetic wave reflected from the sample. During the experiments, the samples were placed in a hole dug in the absorbing material, which 
can reduce reflections from the environment. The humidity and temperature of the room remained constant during laboratory testing (the air temperature was 23 degrees Celsius and the relative humidity was 45-55\%). During the test, 1000 points were scanned in the vector network analyzer in the $90-140 \mathrm{GHz}$ band, and the test error was not more than 0.05 GHz. Additionally, the numerical results for the resonant frequency points obtained from the FEM were fitted to the experimental measurement results by changing the dielectric constant of LC layer in the computational model. Thus, the equivalent dielectric constant of LC layer was obtained [26].
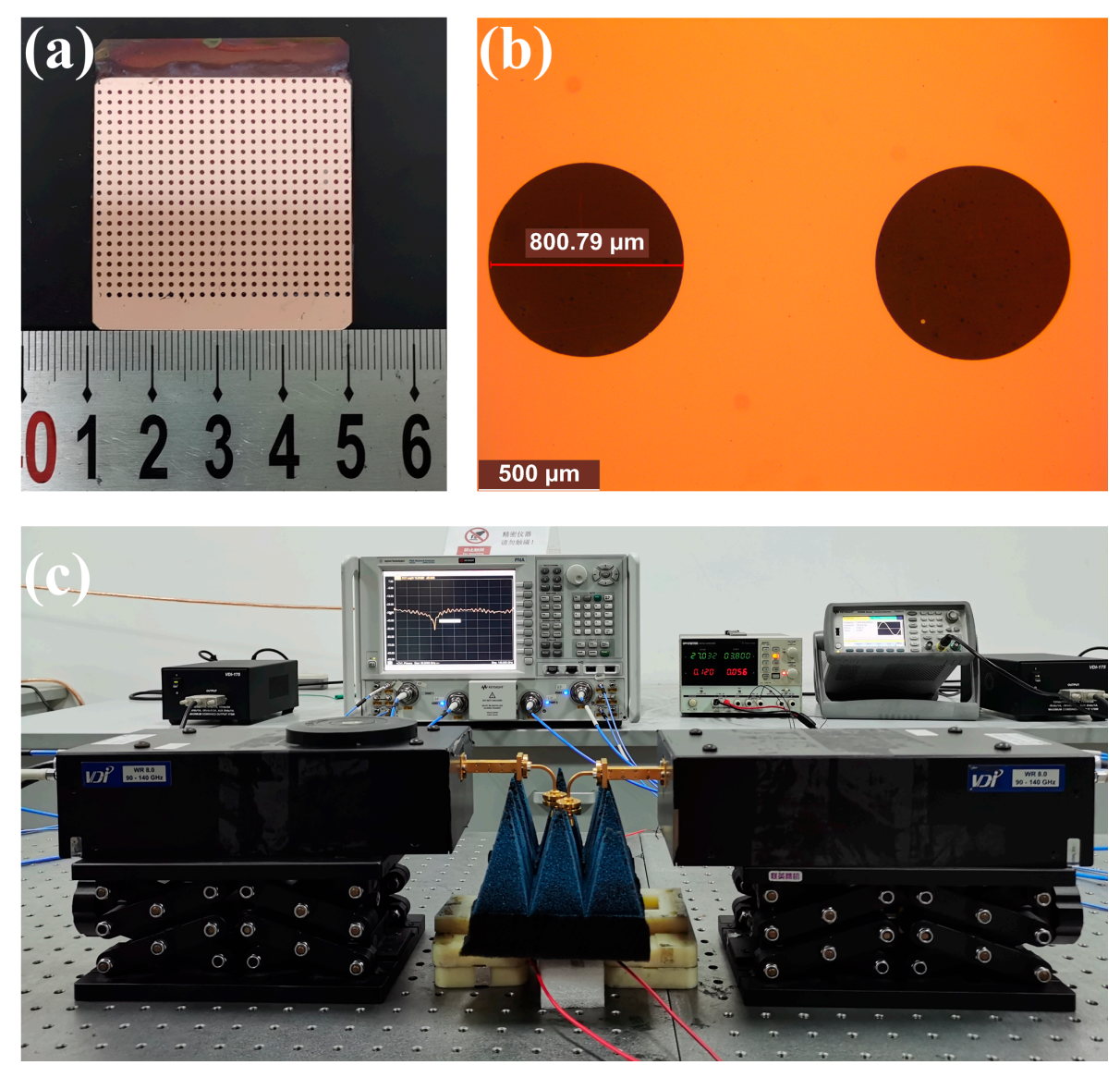

Figure 2. (a) The sample structure $\left(4 \times 4 \mathrm{~cm}^{2}\right)$. (b) The unit cells of the periodic pattern under a metallographic microscope. (c) Sample testing apparatus.

Figure 3 shows the simulation and experimental results obtained for the S200 LC (Shijiazhuang Chengzhi Yong Hua Display Materials Co., Ltd.) with $h l c=30,35,40$, 45, 50, 60,65 , and $100 \mu \mathrm{m}$. When the thickness of the LC layer is $30 \mu \mathrm{m}$, the maximum shift range of the resonant frequency is $8.15 \mathrm{GHz}$. The resonant frequency of the FSS shifts toward lower frequencies (from 108.4 to $105.4 \mathrm{GHz}$ ) as the thickness of the LC layer increases from 30 to $100 \mu \mathrm{m}$ without any bias voltage. On the other hand, when the applied bias voltage reaches saturation, the resonant frequency remains almost constant at around $100 \mathrm{GHz}$. Since the LC molecules under the hollow circular region of the patterned copper layer cannot be completely reoriented, the maximum error at the resonant frequency point is about $0.91 \%$, and the corresponding LC layer equivalent dielectric constants error is about $2 \%$ [29]. In addition, we also considered the influence of hollow circle radius errors caused by manufacturing. In this study, the hollow-circle radii measured by metallographic microscope were used for the fitting of the equivalent dielectric constants, so the errors due to the hole radius were very small. It can also be seen from the figures that each resonant frequency on the FSS features a one-to-one correspondence with the equivalent dielectric 
constant of the LC layer; this renders it possible to calculate the equivalent dielectric constant of the LC layer by fitting the
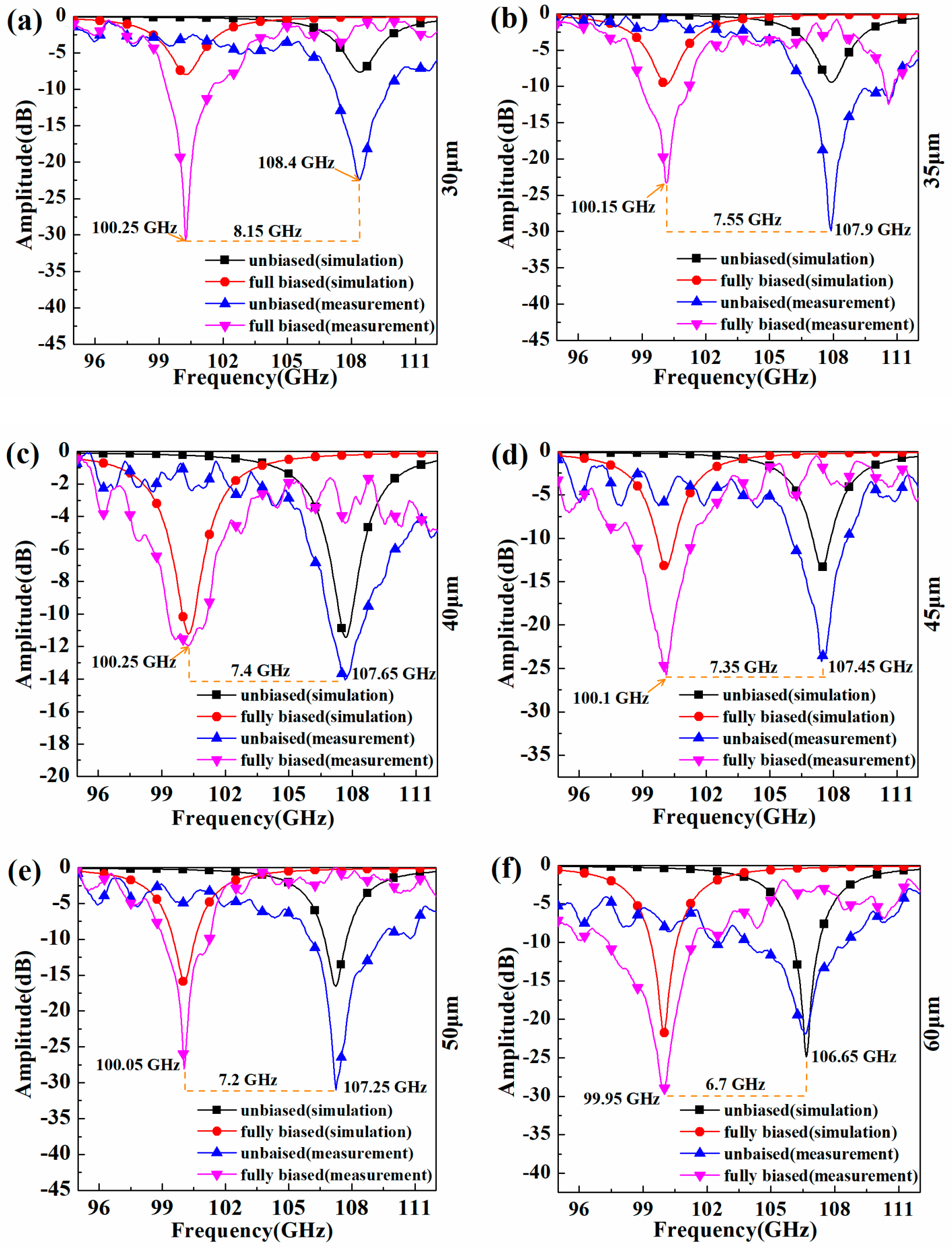

Figure 3. Cont. 

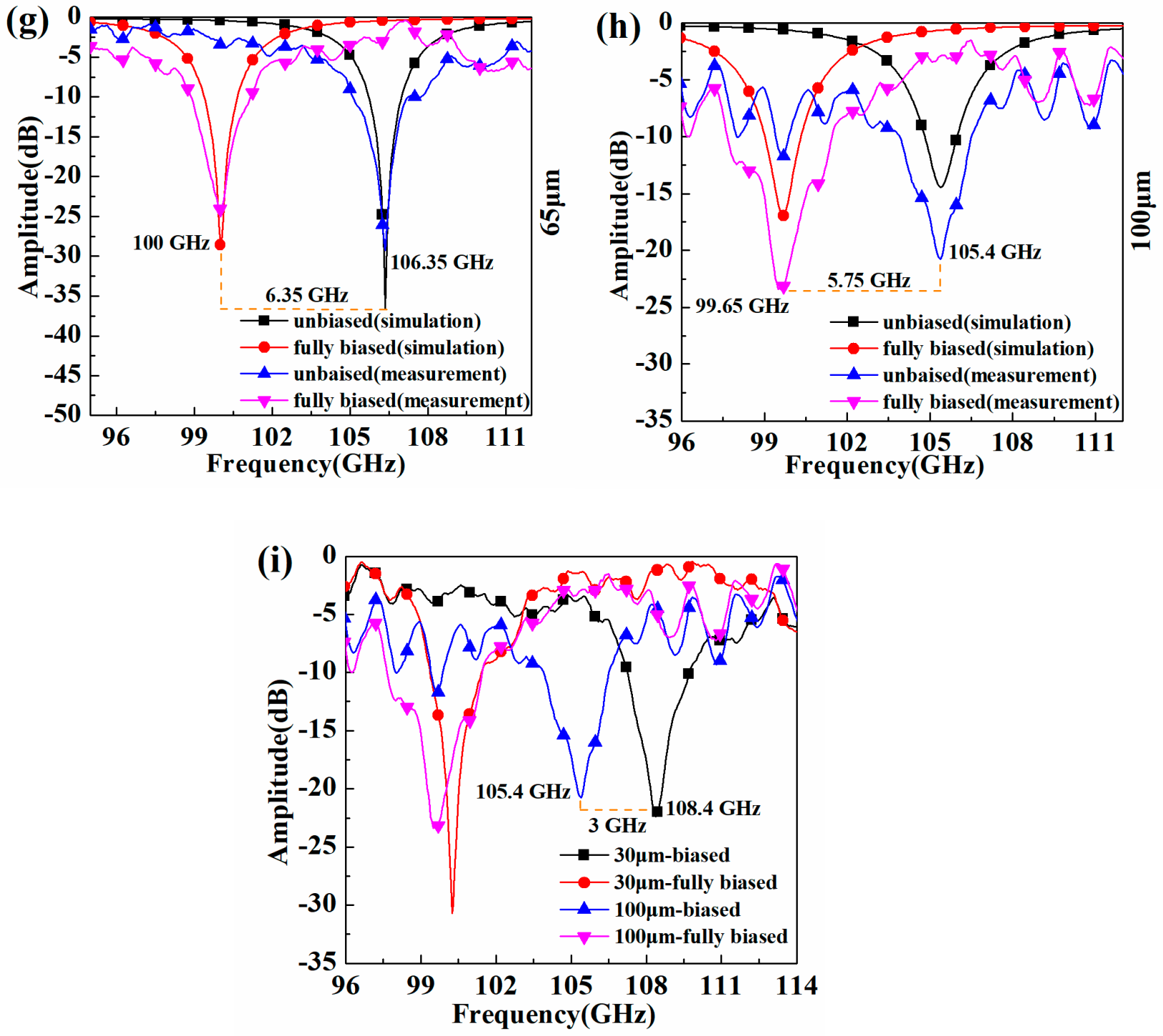

Figure 3. With different thicknesses: (a) $h l c=30 \mu \mathrm{m},(\mathbf{b}) h l c=35 \mu \mathrm{m}$, (c) $h l c=40 \mu \mathrm{m}$, (d) $h l c=45 \mu \mathrm{m}$, (e) $h l c=50 \mu \mathrm{m},(\mathbf{f}) h l c=60 \mu \mathrm{m},(\mathrm{g}) h l c=65 \mu \mathrm{m}$, and (h) $h l c=100 \mu \mathrm{m}$. (i) Measured reflection spectra at 30 and $100 \mu \mathrm{m}$.

As shown in Figure 4 , the dielectric properties $\left(\varepsilon_{\perp}, \varepsilon / /, \Delta \varepsilon\right)$ of the LC layers with different thicknesses are determined by fitting the numerical simulation results to the experimental data. We fitted the simulated reflection spectra to the experimental spectra at different voltages and obtain the values of the equivalent dielectric constant of the LC layer. Table 2 lists the dielectric properties obtained. The results show that the initial equivalent dielectric constant $\left(\varepsilon_{\perp}\right)$ of the LC layer increases with the increase in the thickness of the LC layer, while the saturation equivalent dielectric constant $(\varepsilon / /)$ of the LC layer remains almost constant. The reason for this behavior is that the initial state of the LC layer mainly depends on the role of the alignment layer. As the thickness of the LC layer gradually increases, the force exerted by the alignment layer on the LC intermediate layer becomes smaller. A component of the long axis of some branches in the intermediate layer lies along the vertical direction (i.e., the z-axis direction); thus, the equivalent $\varepsilon_{\text {eff } \perp}$ increases. As the LC layer thickness increases from 30 to $100 \mu \mathrm{m}, \varepsilon_{\text {eff } \perp}$ increases from 2.56 to 2.65 . 


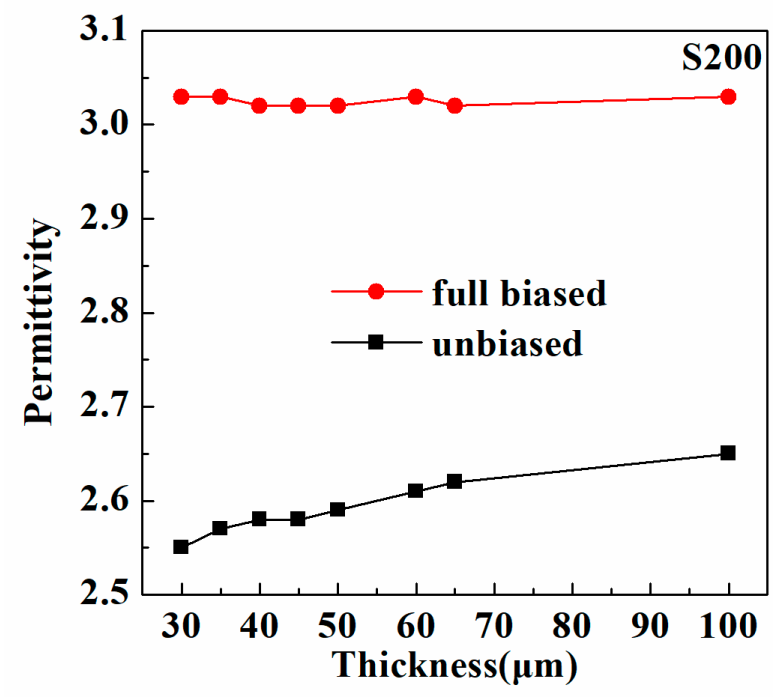

Figure 4. Permittivity as a function of the LC layer thickness for the LC S200 in the fully biased and unbiased states.

Table 2. Dielectric properties of the S200 LC with different thicknesses.

\begin{tabular}{cccccccccc}
\hline Thickness $(\boldsymbol{\mu m})$ & $\mathbf{3 0}$ & $\mathbf{3 5}$ & $\mathbf{4 0}$ & $\mathbf{4 5}$ & $\mathbf{5 0}$ & $\mathbf{6 0}$ & $\mathbf{6 5}$ & $\mathbf{1 0 0}$ \\
\hline$\varepsilon_{\perp}$ & 2.55 & 2.57 & 2.58 & 2.58 & 2.59 & 2.61 & 2.62 & 2.65 \\
\hline$\varepsilon_{/ /}$ & 3.03 & 3.03 & 3.02 & 3.02 & 3.02 & 3.03 & 3.02 & 3.03 \\
\hline$\Delta \varepsilon$ & 0.48 & 0.46 & 0.44 & 0.44 & 0.43 & 0.42 & 0.40 & 0.38 \\
\hline
\end{tabular}

Figure 5 shows the simulation and experimental results obtained for the E7 LC (Jiangsu Hecheng New Material Co. Ltd., Nanjing, China) with $h l c=30,45,60$, and $100 \mu \mathrm{m}$. The LC layers with thicknesses of 30,45, 60, and $100 \mu \mathrm{m}$ result in a frequency-shifting range of 6.3, 5.9, 5.5, and $4.9 \mathrm{GHz}$, respectively. As shown in Figure 6, the equivalent dielectric constant is used as a function of the LC layer thickness in two states. Table 3 lists the values of the dielectric properties $\left(\varepsilon_{\perp}, \varepsilon_{/ /}, \Delta \varepsilon\right)$ of E7. However, E7 exhibits a small change in the equivalent dielectric constant within the thickness range of 30-100 $\mu \mathrm{m}$, which indicates that the alignment induced by $\mathrm{Pi}$ is also closely related to the $\mathrm{LC}$ molecular structure. Further investigations on thicker LC layers will be conducted in the future.
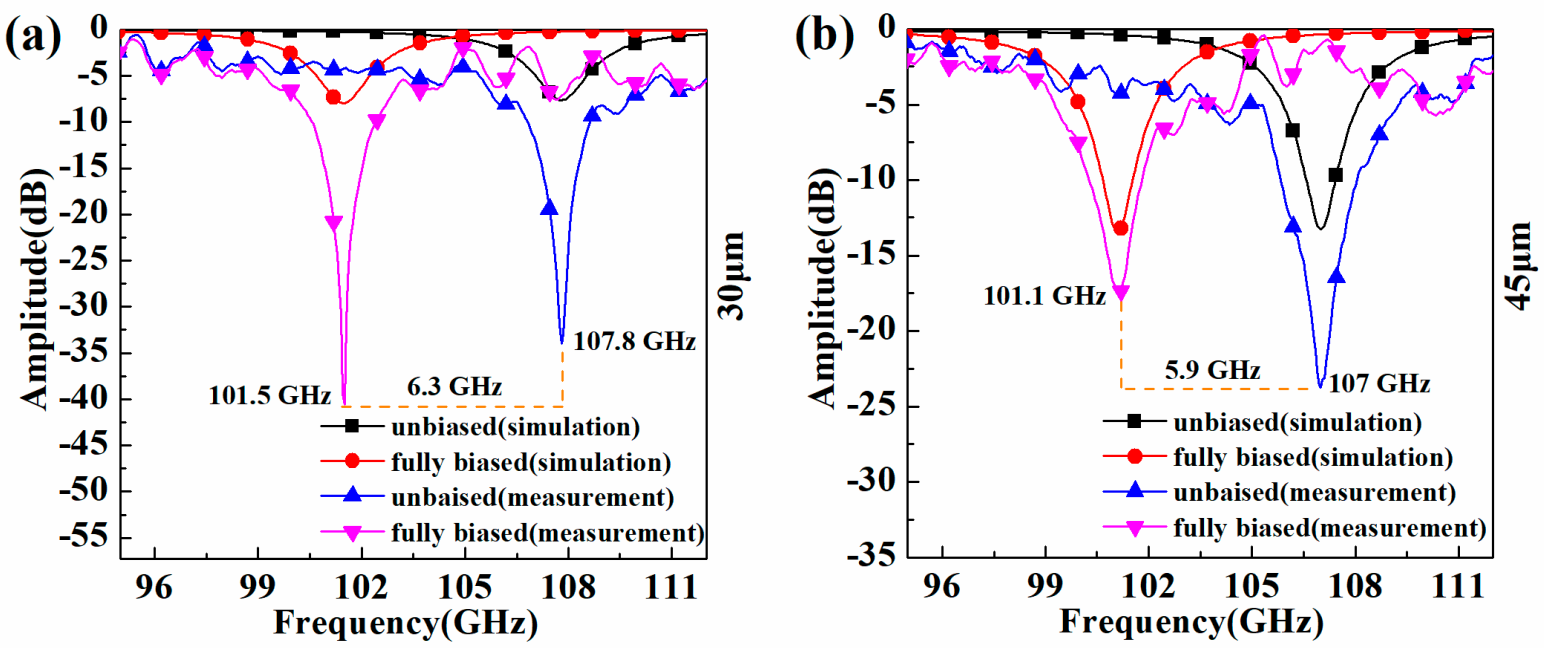

Figure 5. Cont. 

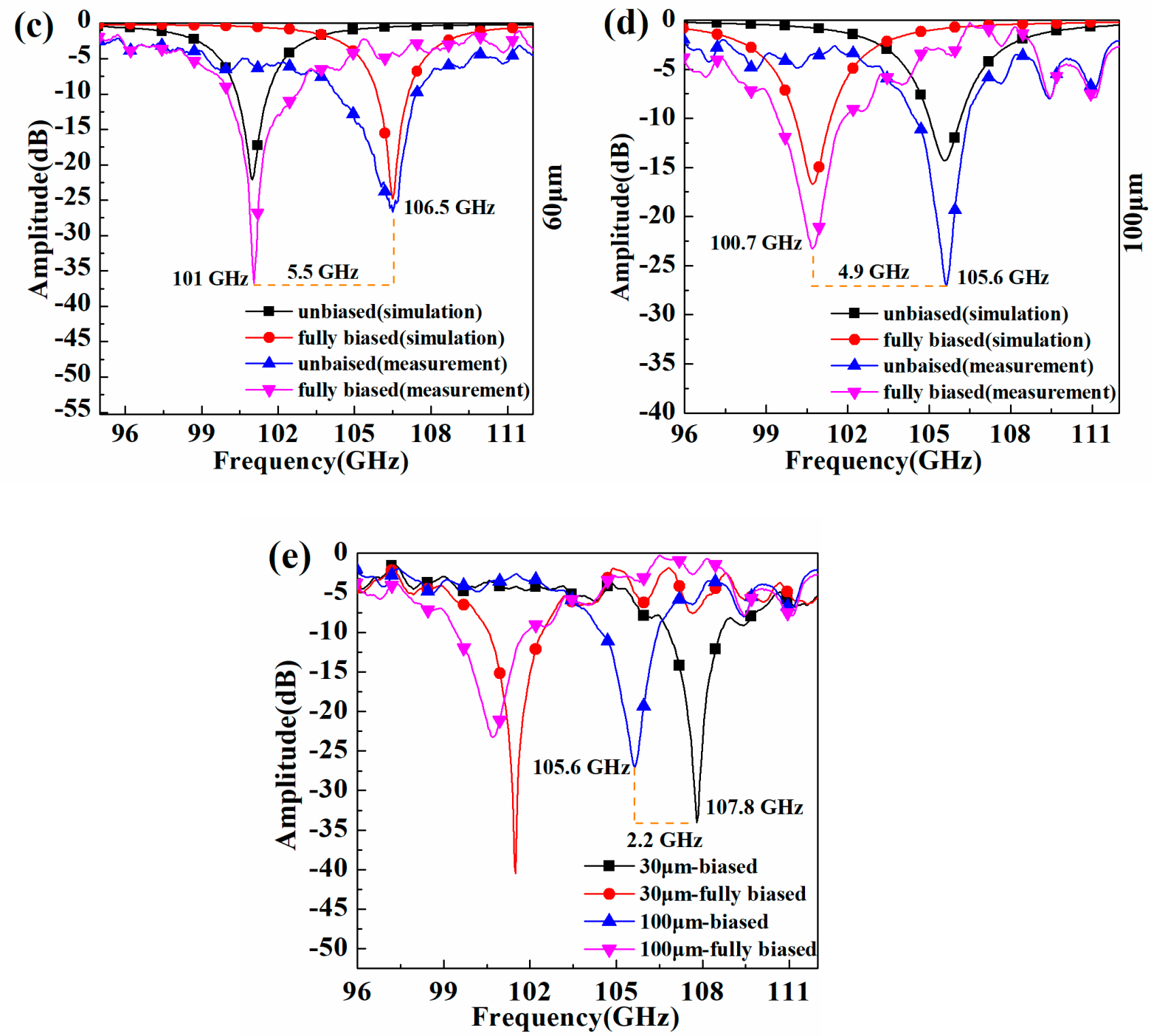

Figure 5. With different thicknesses: (a) $h l c=30 \mu \mathrm{m}$, (b) $h l c=45 \mu \mathrm{m}$, (c) $h l c=60 \mu \mathrm{m}$ and (d) $h l c=100 \mu \mathrm{m}$. (e) Measured reflection spectra at 30, 45, 60, and $100 \mu \mathrm{m}$.

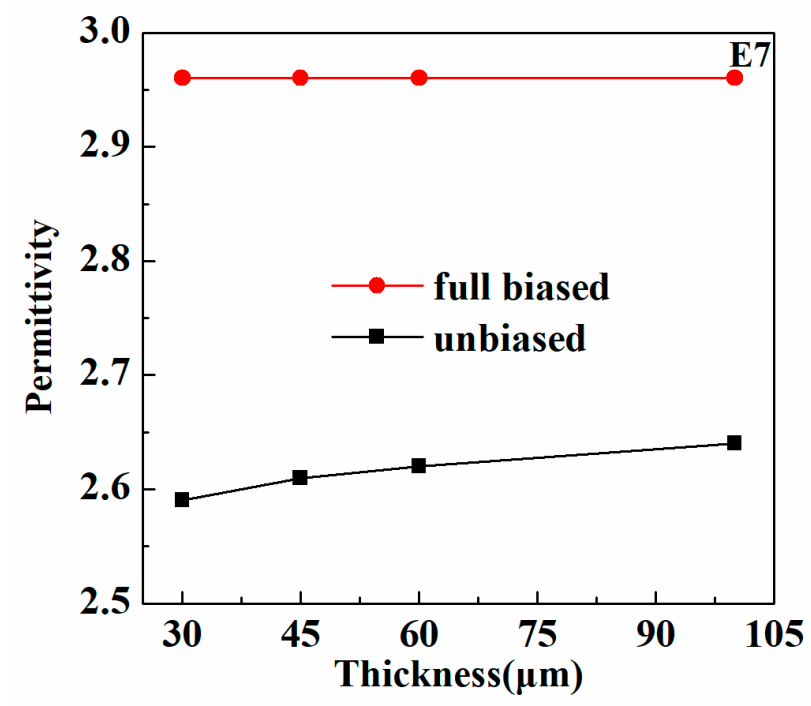

Figure 6. Permittivity as a function of the LC layer thickness for the LC E7 in the fully biased and unbiased states. 
Table 3. Dielectric properties of the E7 LC with different thicknesses.

\begin{tabular}{ccccc}
\hline Thickness $(\boldsymbol{\mu m})$ & $\mathbf{3 0}$ & $\mathbf{4 5}$ & $\mathbf{6 0}$ & $\mathbf{1 0 0}$ \\
\hline$\varepsilon_{\perp}$ & 2.59 & 2.61 & 2.62 & 2.64 \\
\hline$\varepsilon_{/ /}$ & 2.96 & 2.96 & 2.96 & 2.96 \\
\hline$\Delta \varepsilon$ & 0.37 & 0.35 & 0.34 & 0.32 \\
\hline
\end{tabular}

\section{Conclusions}

For the design of LC-based tunable devices, it is necessary to systematically understand the characteristics of LC materials; thus, measuring the dielectric properties of LC materials is important. The effect of the LC layer thickness on the equivalent dielectric constant was analyzed in this study. We varied the thickness of the LCs S200 and E7 and measured their dielectric properties in the 90 to $140 \mathrm{GHz}$. We found that the initial equivalent dielectric constant increases when the thickness of both LC layers increases, while the saturation equivalent dielectric constant of the LC layer remains essentially unchanged. Our work provides relevant information regarding the dielectric properties of LC materials for their application in infrared, $\mathrm{GHz}$, and $\mathrm{THz}$ bands, so as to better design and optimize tunable devices.

Author Contributions: Conceptualization, R.M. and J.Y.; methodology, G.Z.; software, R.M. and L.X.; validation, R.M., G.Z. and L.X.; formal analysis, H.G.; investigation, G.Z; resources, L.X.; data curation, R.M.; writing —original draft preparation, R.M.; writing—review and editing, J.Y.; visualization, Z.Y.; supervision, G.D.; project administration, H.L.; funding acquisition, Z.Y. and J.Y. All authors have read and agreed to the published version of the manuscript.

Funding: This work was supported in part by the Opening Project of Guangxi Key Laboratory of Wireless Wideband Communication and Signal Processing under Grant GXKL06200207 and in part by the National Natural Science Foundation of China under Grant 61871171.

Institutional Review Board Statement: Not applicable.

Informed Consent Statement: Not applicable.

Data Availability Statement: The data presented in this study are available on request from the corresponding author.

Conflicts of Interest: The authors declare no competing financial interest.

\section{References}

1. Wang, L.; Xiao, R.-W.; Ge, S.-J.; Shen, Z.-X.; Lu, P.; Hu, W.; Lu, Y.-Q. Research progress of terahertz liquid crystal materials and devices. Acta Phys. Sin. 2019, 68, 084205. [CrossRef]

2. Chen, Z.-Z.; Zhao, H.-Z.; Li, M.; Bu, J.; Ma, H. Application and research progress of liquid crystal materials in THz band. Electron. Compon. Mater. 2015, 34, 1-4.

3. Parka, J.; Sielezin, K. 0.3-10.0 THz spectra for chosen liquid crystal molecules-Simulation and physical properties. Mol. Cryst. Liq. Cryst. 2017, 657, 66-71. [CrossRef]

4. Wu, J.-B.; Shen, Z.; Ge, S.-J.; Chen, B.-W.; Shen, Z.-X.; Wang, T.-F.; Zhang, C.-H.; Hu, W.; Fan, K.-B.; Padilla, W.; et al. Liquid crystal programmable metasurface for terahertz beam steering. Appl. Phys. Lett. 2020, 116, 131104. [CrossRef]

5. Wang, S.; Li, Q.; Gu, J.-Q.; Han, J.-G.; Zhang, W.-L. Dielectric properties of MgO-ZnO-TiO2-based ceramics at $1 \mathrm{MHz}$ and THz frequencies. J. Mater. Sci. 2017, 52, 9335-9343. [CrossRef]

6. Wang, J.; Tian, H.; Wang, Y.; Li, X.-Y.; Cao, Y.-J.; Li, L.; Liu, J.-L.; Zhou, Z.-X. Liquid crystal terahertz modulator with plasmoninduced transparency metamaterial. Opt. Express 2018, 26, 5769-5776. [CrossRef]

7. Zhang, L.-H.; Li, J.-J.; Bao, Y.-H.; Wang, A.-X.; Zhang, W.-G. A broad stop-band filter based on multilayer metamaterials in the THz regime. J. Infrared Millim. Waves 2016, 35, 267-270.

8. Zhou, S.-H.; Shen, Z.-X.; Kang, R.-Y.; Ge, S.-J.; Hu, W. Liquid Crystal Tunable Dielectric Metamaterial Absorber in the Terahertz Range. Appl. Sci. 2018, 8, 2211. [CrossRef]

9. Kowerdziej, R.; Olifierczuk, M.; Salski, B.; Parka, J. Tunable negative index metamaterial employing in-plane switching mode at terahertz frequencies. Liq. Cryst. 2017, 39, 827-831. [CrossRef]

10. Kowerdziej, R.; Stanczyk, T.; Parka, J. Electromagnetic simulations of tunable terahertz metamaterial infiltrated with highly birefringent nematic liquid crystal. Liq. Cryst. 2015, 42, 430-434. [CrossRef] 
11. Qiu, Q.-L.; Yu, X.-P.; Sui, W.-Q. A K-Band Low-Power Phase Shifter Based on Injection Locked Oscillator in $0.13 \mathrm{mu} \mathrm{m}$ CMOS Technology. J. Infrared Millim. Terahertz Waves 2017, 38, 1368-1386. [CrossRef]

12. Ma, S.; Wang, P.-Y.; Meng, F.-Y.; Fu, J.-H.; Wu, Q. Electronically controlled beam steering leaky wave antenna in nematic liquid crystal technology. Int. J. RF Microw. Comput.-Aid. Eng. 2020, 30, e22188. [CrossRef]

13. James, R.; Fernandez, F.A.; Day, S.E.; Bulja, S.; Mirshekar-Syahkal, D. Characterisation and Applications of Nematic Liquid Crystals in Microwave Devices. Mol. Cryst. Liq. Cryst. 2011, 542, 196-203. [CrossRef]

14. Srivastava, A.K.; Misra, A.K.; Shukla, J.P.; Manohar, R. Dielectric and electro-optical properties of dye doped ferroelectric liquid crystal. Phys. Lett. A 2008, 372, 6254-6259. [CrossRef]

15. Yang, F.-Z.; Sambles, J.R. Determination of the permittivity of nematic liquid crystals in the microwave region. Liq. Cryst. 2003, 30, 599-602. [CrossRef]

16. Liu, Y.-R.; Shen, J.-R.; Shen, T.; Zheng, J.-H.; Zhuang, S.-L. Electro-optical properties and frequency response of polymer-dispersed liquid crystal gratings doped with multi-walled carbon nanotubes. J. Mater. Sci. 2021, 56, 12660-12670. [CrossRef]

17. Aizen, A.; Abdulhalim, I. Dielectric permittivity imaging based on a liquid crystal capacitive sensor. Liq. Cryst. XXI 2017, 10361, 103610U.

18. Yazdanpanahi, M.; Bulja, S.; Mirshekar-Syahkal, D.; James, R.; Day, S.E.; Fernandez, F.A. Measurement of Dielectric Constants of Nematic Liquid Crystals at mm-Wave Frequencies Using Patch Resonator. IEEE Trans. Instrum. Meas. 2010, 59, $3079-3085$. [CrossRef]

19. Dickie, R.; Baine, P.; Cahill, R.; Doumanis, E.; Goussetis, G.; Christie, S.; Mitchell, N.; Fusco, V.; Linton, D.; Encinar, J.; et al. Electrical characterisation of liquid crystals at millimetre wavelengths using frequency selective surfaces. Electron. Lett. 2012, 48, 611-612. [CrossRef]

20. Deo, P.; Mirshekar-Syahkal, D.; Seddon, L.; Day, S.E.; Fernandez, F.A. Microstrip Device for Broadband (15-65 GHz) Measurement of Dielectric Properties of Nematic Liquid Crystals. IEEE Trans. Microw. Theory Tech. 2015, 63, 1388-1398. [CrossRef]

21. Ji, Y.-Y.; Fan, F.; Zhang, X.; Cheng, J.-R.; Chang, S.-J. Terahertz birefringence anisotropy and relaxation effects in polymer-dispersed liquid crystal doped with gold nanoparticles. Opt. Express 2020, 28, 17253-17265. [CrossRef] [PubMed]

22. Johri, M.; Tiwari, A.; Saxena, S.; Johri, S.; Kumar, D.; Singh, D.P. Study of the dielectric response and thermo-dynamical properties of PentylCyano Biphenyl (PCB) Liquid Crystal using microwave cavity spectrometer as a probe. Measurement 2020, 166, 108156. [CrossRef]

23. Nova, V.; Bachiller, C.; Villacampa, B.; Kronberger, R.; Boria, V.E. Characterization of Nematic Liquid Crystals at Microwave Frequencies. Crystals 2020, 10, 1106. [CrossRef]

24. Perez-Palomino, G.; Florencio, R.; Encinar, J.A.; Barba, M.; Dickie, R.; Cahill, R.; Baine, P.; Bain, M.; Boix, R.R. Accurate and Efficient Modeling to Calculate the Voltage Dependence of Liquid Crystal-Based Reflectarray Cells. IEEE Trans. Antennas Propag. 2014, 62, 2659-2668. [CrossRef]

25. Gao, S.; Yang, J.; Wang, P.; Zheng, A.-D.; Lu, H.-B.; Deng, G.-S.; Lai, W.-E.; Yin, Z.-P. Tunable Liquid Crystal Based Phase Shifter with a Slot Unit Cell for Reconfigurable Reflectarrays in F-Band. Appl. Sci. 2018, 8, 2528. [CrossRef]

26. Deshmukh, R.R.; Malik, M.K. Effect of temperature on the optical and electro-optical properties of poly(methyl methacrylate)/E7 polymer-dispersed liquid crystal composites. J. Appl. Polym. Sci. 2008, 109, 627-637. [CrossRef]

27. Vieweg, N.; Shakfa, M.K.; Scherger, B.; Mikulics, M.; Koch, M. THz Properties of Nematic Liquid Crystals. J. Infrared Millim. Terahertz Waves 2010, 31, 1312-1320. [CrossRef]

28. Park, H.; Parrott, E.P.J.; Fan, F.; Lim, M.; Han, H.; Chigrinov, V.G.; Pickwell-MacPherson, E. Evaluating liquid crystal properties for use in terahertz devices. Opt. Express 2012, 20, 11899-11905. [CrossRef]

29. Lu, H.-B.; Jing, S.-C.; Xia, T.-Y.; Yang, J.; Yin, Z.-P.; Deng, G.-S. Measurement of LC dielectric constant at lower terahertz region based on metamaterial absorber. IEICE Electron Express 2017, 14, 2017046. [CrossRef]

30. Tosaka, T.; Fujii, K.; Fukunaga, K.; Kasamatsu, A. Development of Complex Relative Permittivity Measurement System Based on Free-Space in 220-330-GHz Range. IEEE Trans. Terahertz Sci. Technol. 2015, 5, 102-109. [CrossRef] 